\title{
Ultrasonographic evaluation of acute pelvic pain in pregnant and postpartum period
}

\author{
Sung Bin Park'1, Byoung Hee Han², Young Ho Lee²
}

${ }^{1}$ Department of Radiology, Chung-Ang University Hospital, Chung-Ang University College of Medicine, ${ }^{2}$ Department of Radiology, Cheil General Hospital \& Women's Healthcare Center, Dankook University College of Medicine, Seoul, Korea

\begin{abstract}
Acute pelvic pain in pregnant and postpartum patients presents diagnostic and therapeutic challenges. The interpretation of imaging findings in these patients is influenced by the knowledge of the physiological changes that occur during the pregnant and postpartum period, as well as by the clinical history. Ultrasonography remains the primary imaging investigation of the pregnant and postpartum women. This article describes the causes and imaging features of acute pelvic pain in pregnant and postpartum period and suggests characteristics to such diseases, focusing on the ultrasonography features that allow one to arrive at the corrective diagnosis. Knowledge of the clinical settings and imaging features of acute pelvic pain in pregnant and postpartum period can lead to accurate diagnosis and appropriate management of the condition.
\end{abstract}

Keywords: pelvic pain, ultrasonography, obstetric, pregnancy, postpartum period

Acute pelvic pain (APP) (defined as noncyclic intense pain characterized by sudden onset lasting for less than 3 months) and vaginal bleeding are two of the most common presenting symptoms of premenopausal women in obstetrics and gynecologic emergency departments or physicians' offices [1-3]. APP is a nonspecific symptom and there is a broad spectrum of obstetric, gynecologic and other causes, including gastrointestinal, urologic, and musculoskeletal etiologies [4]. For pregnant and postpartum patients, the diagnostic approach is often more challenging from the unhelpfulness of clinical examinations and laboratory tests [5]. The diagnosis of APP in pregnant women is confounded by several factors found in a normal pregnancy, such as nonspecific leukocytosis, displacement

Received 17.08.2016 Accepted 23.10.2016

Med Ultrason

2017, Vol. 19, No 2, 218-223

Corresponding author: Sung Bin Park,

Department of Radiology, Chung-Ang

University Hospital, Chung-Ang University

College of Medicine,

102, Heukseok-ro, Dongjak-gu,

Seoul, 06973, Korea

Phone: 82-2-6299-3196, Fax: 82-2-6263-1557

E-mail: pksungbin@paran.com of abdominal and pelvic structures from their normal locations by the gravid uterus, a difficult clinical examination with often-delayed peritoneal signs by laxity of the anterior abdominal wall, and nonspecific nausea and vomiting [5-8]. Therefore, imaging is frequently required for differential diagnosis, in addition to the patient's clinical history, physical examination, and laboratory data.

Ultrasonography (US) is the imaging modality of choice for diagnosing maternal-related abnormalities both during and following pregnancy and delivery because of its availability, portability, and lack of ionizing radiation $[2,5,9]$. US plays a major role in such cases as the most important tools to identify the correct etiology and diagnosis [9]. US may be also useful in follow-up. Computed tomography (CT) is being used more frequently in the emergency evaluation of the female patient with acute pelvic pain as its use is becoming more widespread and physicians become more familiar with it $[3,10]$. Although CT has ionizing radiation and is not usually appropriate for pregnant patients, it is more useful and serves an important role in patients with postpartum APP [10]. Magnetic resonance (MR) imaging may also be appropriate in the evaluation of acute pelvic pain in pregnancy when the US is indeterminate or for 
patients who require a wider search beyond the field of view available with US $[3,11]$.

APP can be further categorized into obstetric and nonobstetric causes [4]. The first step in the evaluation of premenopausal women with acute pelvic pain is to establish if the patient is pregnant, by serum $\beta$-human chorionic gonadotropin (hCG) levels in correlation with the menstrual history $[1,12,13]$. The next step is to group lesions according to their anatomic locations, physiological or pathological origin, and internal characteristics (cystic, solid, or complex cystic) for an efficient and accurate diagnosis [13]. Some causes are unique to pregnancy, are exacerbated by pregnancy, or require an altered imaging algorithm for diagnosis during pregnancy.
In this paper we categorized the APP in the pregnant (table I) and postpartum period (table II). We also described imaging features focusing on the US features.

\section{Acute pelvic pain in pregnant period}

\section{Complicated corpus luteal cyst}

A corpus luteum is the remnant of a mature ovarian follicle after ovulation and is usually seen during the secretory phase of the menstrual cycle or the $1^{\text {st }}$ trimester during pregnancy [1]. If a patient becomes pregnant, the corpus luteum secretes progesterone and maintains the pregnancy until the placenta forms [1]. Either the failure

Table I. Summary of acute pelvic pain in the pregnant period

\begin{tabular}{|c|c|}
\hline Disease & Characteristics and Differential Diagnosis Points \\
\hline Corpus luteal cyst & $\begin{array}{l}\text { Seen 1st trimester of pregnancy; usually, spontaneous regression } \\
\text { Internal hemorrhage, leaking, or rupture cause acute pelvic pain } \\
\text { Homogeneous thick moderately echogenic wall } \\
\text { High vascularity with a low resistance arterial waveform } \\
\text { Frequently associated with hemorrhage: low-level echogenicity in a fine, lace-like, reticular pattern }\end{array}$ \\
\hline Ectopic pregnancy & $\begin{array}{l}\text { Reproductive age women with acute pelvic pain and positive serum } \beta \text {-hCG levels } \\
\text { Most common site: ampullary portion of fallopian tube } \\
\text { Uncommon site: other areas of fallopian tube, cervix, myometrial scar, ovary, and peritoneal cavity } \\
\text { Quantitative measurement of serum } \beta \text {-hCG levels and transvaginal US are essential and should be fol- } \\
\text { lowed closely } \\
\text { Endometrial finding: pseudogestational sac sign, } 10 \% \text { to } 20 \% \\
\text { Adnexal findings: extrauterine gestational sac, tubal ring, tubal mass } \\
\text { Pelvic hemorrhage } \\
\text { Differential diagnosis from corpus luteal cyst: more echogenic wall, moves separately from ovary, both } \\
\text { low and high resistive index }\end{array}$ \\
\hline $\begin{array}{l}\text { Failed intrauterine } \\
\text { pregnancy }\end{array}$ & $\begin{array}{l}\text { Pregnancy loss or termination before } 20 \text { weeks of gestational age, majority occur during the first } 16 \text { weeks } \\
\text { Criteria of pregnancy failure: } \\
\text {-mean gestational sac diameter } \geq 25 \mathrm{~mm} \text {, no visible embryo } \\
\text {-embryo size } \geq 7 \mathrm{~mm} \text {, no visible heart beat } \\
\text {-gestational sac with/without yolk sac, no visible heart bear in } 11 / 14 \text { days } \\
\text { Cervical incompetence in } 2 \text { nd trimester }\end{array}$ \\
\hline $\begin{array}{l}\text { Gestational tropho- } \\
\text { blastic disease }\end{array}$ & $\begin{array}{l}\text { Spectrum including hydatidiform mole, invasive mole, and choriocarcinoma } \\
\text { First-trimester bleeding and pain } \\
\text { Abnormal proliferation of trophoblastic tissue with excessive } \beta \text {-hCG } \\
\text { Heterogeneous echogenic endometrial mass with multiple variable-sized small anechoic cysts, "cluster of } \\
\text { grapes" } \\
\text { Increased vascularity with low resistance waveforms }\end{array}$ \\
\hline Placental abruption & $\begin{array}{l}\text { Bleeding along the basal plate of the placenta, separating from uterine wall } \\
\text { Increased risk of preterm delivery and fetal death } \\
\text { Subchorionic or retroperitoneal hematoma } \\
\text { Diagnostic performance of US is low }\end{array}$ \\
\hline $\begin{array}{l}\text { Ovarian hyperstimu- } \\
\text { lation syndrome }\end{array}$ & $\begin{array}{l}\text { Ovulation induction, ascites } \\
\text { Marked enlargement of both ovaries } \\
\text { Multiple, large, peripherally located, thin-walled cysts }\end{array}$ \\
\hline $\begin{array}{l}\text { Hyperreactio lute- } \\
\text { inalis }\end{array}$ & $\begin{array}{l}\text { Intrinsic causes such as gestational trophoblastic disease, triplet pregnancies } \\
\text { No ovulation induction } \\
\text { Relatively uniform size of each locules }\end{array}$ \\
\hline $\begin{array}{l}\text { Red or hemorrhagic } \\
\text { degeneration of uter- } \\
\text { ine leiomyoma }\end{array}$ & $\begin{array}{l}\text { Tend to enlarge during pregnancy or involute postpartum } \\
\text { Acute pelvic pain can occur when a leiomyoma infarcts or undergoes degeneration } \\
\text { Anechoic cystic spaces and echogenic area }\end{array}$ \\
\hline
\end{tabular}


of the corpus luteum to involute or its hemorrhage may result in cystic enlargement [13].

Most commonly seen on US, the corpus luteum appears as a round anechoic ovarian cystic mass with a homogeneously thick, moderately echogenic wall, which may be highly vascular with a low resistance arterial waveform [1]. Hemorrhage into a corpus luteum can create a sonographic pattern of internal echoes similar to a hemorrhagic follicular cyst. The typical appearance is that of a complex mass with internal echoes and some degree of posterior through-transmission $[13,14]$.

\section{Ectopic pregnancy $(E P)$}

EP results when a blastocyst abnormally implants in a location that is not the endometrium [15]. The possibility of EP should be considered in any woman of reproductive age who presents with acute pelvic pain and a positive $\beta$-hCG level. EPs account for approximately $2 \%$ of all pregnancies $[15,16]$. Risk factors for EP include a history of prior EPs, pelvic inflammatory disease, infertility treatment, prior pelvic surgery, endometriosis, and pregnancy that occurs after placement of an intrauterine device [16,17]. Although patients with EPs can be asymptomatic, EPs more commonly present with acute pelvic pain, vaginal bleeding, and a palpable adnexal mass or peritoneal signs [16].

Evaluation for suspected EP should begin with a quantitative measurement of serum $\beta$-hCG levels and transvaginal US [18]. When neither an intrauterine pregnancy (IUP) nor specific findings of an EP can be documented in a patient with a subthreshold $\beta$-hCG level (a so-called 'pregnancy of unknown location'), the patient should be closely monitored with serial US, and $\beta$-hCG levels should be sequentially measured until either an IUP or an $\mathrm{EP}$ is identified $[15,16]$. To diagnose EP, the presence or absence of an IUP should be initially evaluated. Next, a scan of the most common EP location, the fallopian tube, should be performed. The ovary provides a landmark for the evaluation of the adnexa, as it is usually located near the ampullary portion of the fallopian tube, which is the most frequent site of EP. The area surrounding the ovary or between the ovary and uterus should be evaluated thoroughly. Then, the uncommon EP locations, such as the ovary, cervix, and peritoneal cavity should be scanned.

The most specific or conclusive sonographic finding of an EP is the presence of an extrauterine gestational sac containing a yolk sac with or without an embryo $[15,16]$. The other findings that can be seen with EP are a tubal mass or tubal ring (fig 1a). In terms of the tubal ring, a differential diagnosis from corpus luteal cyst is important. Establishing an intraovarian location strongly favors the diagnosis of a corpus luteal cyst because intraovarian EPs are rare, accounting for less than $1 \%$ of all EPs $[1,16]$. An intraovarian location can be confirmed by the synchronous movement of the structure with the ovary after manual compression of the ovary through a vaginal probe and anterior abdominal wall during the transvaginal US or by the demonstration of a small rim of ovarian tissue surrounding the lesion $[3,16]$.

Table II. Summary of acute pelvic pain in the postpartum period

\begin{tabular}{|c|c|}
\hline Disease & Characteristics and Differential Diagnosis Points \\
\hline Endometritis & $\begin{array}{l}\text { Common cause of postpartum acute pelvic pain and fever } \\
\text { Occurs after cesarean section more commonly than vaginal delivery } \\
\text { Fluid and echogenic debris consistent with hemorrhage or clot } \\
\text { Air in the endometrial cavity: echogenic foci with distal shadowing or ring-down artifacts; concern for } \\
\text { infection, but also visible in healthy postpartum patients }\end{array}$ \\
\hline $\begin{array}{l}\text { Retained products of } \\
\text { conception }\end{array}$ & $\begin{array}{l}\text { Present with vaginal bleeding and acute pelvic pain or cramping } \\
\text { Doppler US may be helpful in differentiating from thrombus } \\
\text { Internal blood flow: low resistance and present at endometrial-myometrial interface }\end{array}$ \\
\hline Ovarian vein thrombosis & $\begin{array}{l}\text { Uncommon, but potentially fatal, postpartum complication. } \\
\text { Critical because of the risk of pulmonary embolism } \\
\text { Hypoechoic tubular or serpiginous avascular structure in the region of the right adnexa and inferior } \\
\text { vena cava } \\
\text { Lack of flow on color Doppler US }\end{array}$ \\
\hline Bladder flap hematoma & $\begin{array}{l}\text { Occurs in patients after cesarean section } \\
\text { Solid complex mass between the posterior bladder wall and anterior wall of the lower uterine segment } \\
\text { Hematomas can also occur in the rectus sheath }\end{array}$ \\
\hline $\begin{array}{l}\text { Uterine dehiscence and } \\
\text { rupture }\end{array}$ & $\begin{array}{l}\text { Unusual complication of vaginal delivery } \\
\text { Women who have had a prior cesarean section } \\
\text { Dehiscence: limited uterine rupture to the cesarean scar with an intact overlying serosal layer of the } \\
\text { uterine wall } \\
\text { Protruding portion of the amniotic sac, an endometrial or myometrial defect, an extrauterine hema- } \\
\text { toma, and hemoperitoneum }\end{array}$ \\
\hline
\end{tabular}




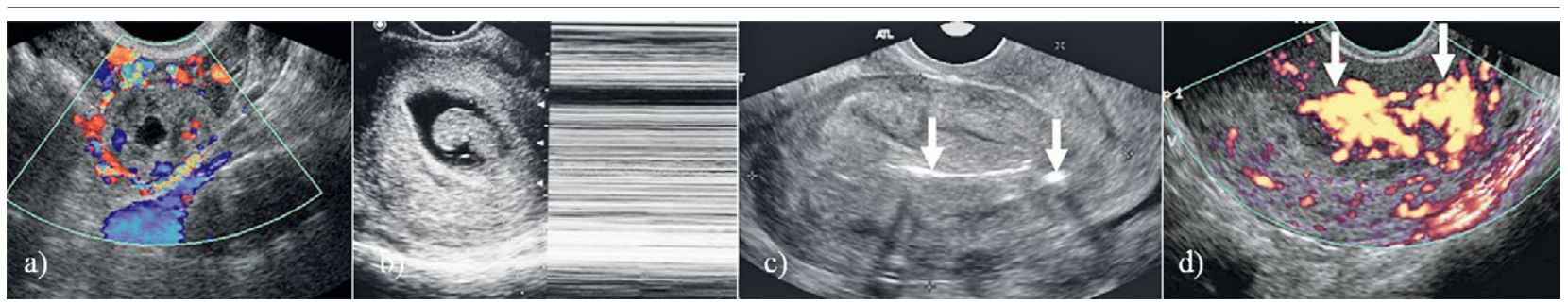

Fig 1. a) Adnexal finding of EP. Transvaginal US of right adnexa shows an adnexal mass with a hyperechoic tubal ring between the ovary and the uterus (not shown). Color Doppler US image shows peripheral hypervascularity. b) Pregnancy failure. Transvaginal US image of the uterus including M-mode scanning demonstrates an embryo (region of interest) larger than $7 \mathrm{~mm}$ without cardiac activity. c) Endometritis. Transvaginal US image of the uterus shows a thick, heterogeneous, endometrial stripe consistent with the presence of debris, hemorrhage, or purulent materials. The outer margins of the endometrial stripe are indistinct. Echogenic areas (arrows) with posterior shadowing and ring-down artifact is consistent with the presence of air. Enhanced CT image shows a complex cystic mass with air densities (not shown). d) RPOC. Transvaginal US image of the uterus shows a heterogeneous mass-like lesion at the posterior uterine corpus with an indistinct endometrial-myometrial interface (not shown). Power Doppler US shows markedly increased vascularity (arrows). In a patient with vaginal bleeding and a history of pregnancy, these findings are consistent with RPOC.

\section{Failed intrauterine pregnancy (IUP)}

Failed IUP is defined as pregnancy loss or termination before 20 weeks of gestational age, but the majority occur during the first 16 weeks [1]. Causes of failed pregnancy include genetic or fetal causes, structural uterine abnormalities, maternal endocrine causes, immunologic causes and infectious causes [8]. US has an important role in differentiating spontaneous abortion or pregnancy failure from a viable IUP or EP [1].

Criteria to diagnose a pregnancy failure at US should be sufficiently generous to allow for follow-up of either any potential viable pregnancy or clear nonviable pregnancy $[14,16]$. When an embryo is larger than or equal to $7 \mathrm{~mm}$, the heartbeat should be detected [5]. Once the mean sac diameter of an IUP is larger than or equal to $25 \mathrm{~mm}$, the embryo should be seen [5]. Once the gestational sac with/without yolk sac is detected, the embryo with a heartbeat should be seen in 11/14 days (fig 1b) [5]. If not, a failed IUP can be diagnosed.

Cervical incompetence is a common cause of failed IUP in the second trimester, manifesting as painless dilatation of the cervix that leads to preterm labor [2]. The US findings include bulging of the fetal membranes into a widened internal os and shortening of the cervical canal.

\section{Gestational trophoblastic disease}

Gestational trophoblastic disease encompasses a broad spectrum of conditions, includes a hydatidiform mole, invasive mole, choriocarcinoma, and placental site trophoblastic tumor $[11,19]$. US findings include heterogeneous echogenic endometrial mass with multiple variable-sized small anechoic cysts, giving the appearance of a "cluster of grapes" and increased vascularity with low resistance waveforms [19].

\section{Placental abruption}

Bleeding along the basal plate of the placenta, separating it from the underlying uterine wall, may result from primary placental abruption [2]. The clinical diagnosis is usually based on vaginal bleeding, abdominal or pelvic pain, a history of trauma or an otherwise unexplained preterm labor and contractions. The US diagnostic performance for placental abruption is low [20], but US is often performed to visualize the extent of the subchorionic (i.e., between the uterine wall and chorionic membrane) or retroperitoneal (behind the placenta) hematoma. Because of the low sensitivity of US in detecting small hematoma, negative US findings do not rule out the presence of placental abruption.

\section{Ovarian hyperstimulation syndrome (OHSS) and \\ Hyperreactio luteinalis}

High circulating serum $\beta$-hCG levels can cause a marked enlargement of bilateral ovaries, with multiple thecal lutein cysts [21]. In the clinical setting of high $\beta$-hCG levels, the three relevant conditions are gestational trophoblastic disease, OHSS and hyperreactio luteinalis. OHSS is an iatrogenic complication of ovulation induction and occurs during early pregnancy or during the luteal phase. The incidence of severe OHSS ranges from $0.5 \%$ to $5 \%$ $[1,22]$. Alternatively, if associated with intrinsic causes, such as normal pregnancy, gestational trophoblastic disease, polycystic ovarian disease, and triplet pregnancies, the condition is called hyperreactio luteinalis $[21,23]$. The clinical manifestations include abdominal fullness, ascites, and pleural effusion. Most cases spontaneously resolve or result in involution after delivery [23].

US findings in patients with OHSS or hyperreactio luteinalis include markedly enlarged multicystic ovaries. This is commonly mistaken for an ovarian tumor; however, the relatively uniform size of each locule favors 
these conditions over an ovarian tumor [21]. MR imaging provides better visualization of both the primary lesions and the predicted sites for peritoneal implants that may be associated with an ovarian malignancy [21].

\section{Red or hemorrhagic degeneration of uterine}

\section{leiomyoma}

Because of continued estrogen stimulation, uterine leiomyomas tend to enlarge during pregnancy or involute postpartum. With rapid growth, they may outgrow their vascular supply, resulting in hemorrhagic infarction and necrosis. US findings of leiomyomas that have undergone red or hemorrhagic degeneration during pregnancy include heterogeneous or hyperechoic lesions. Later leiomyomas tend to demonstrate anechoic components resulting from cystic necrosis, which allows confirmation of the diagnosis $[2,24]$. US can further confirm a degenerating leiomyomas as the source of pain if the patient experiences pain when the probe is directly placed over the leiomyoma [25]. On MR imaging, it typically exhibits diffuse or peripheral high signal intensity on T1-weighted imaging and variable signal intensity on T2-weighted imaging [26]. The hyperintense rim on T1-weighted imaging may correspond to obstructed veins at the periphery of the mass.

\section{Acute pelvic pain in postpartum period}

\section{Endometritis}

Endometritis is a common cause of postpartum pelvic pain and fever and is usually accompanied by leukocytosis and vaginal discharge $[1,10]$. Risk factors include prolonged labor, premature rupture of membranes, and retained products of conception (RPOCs) [1].

Sonographic appearance of endometritis is variable and nonspecific with overlap in the appearance of the normal postpartum endometrium and RPOCs. The endometrium can appear normal or thickened with increased vascularity. Fluid and echogenic debris consistent with hemorrhage or clot are common findings. Echogenic foci with distal shadowing or ring-down artifacts may be seen in patients with air in the endometrial cavity, which presents a concern for infection (fig 1c) [1,27]. Making a clinical correlation (enlarged tender uterus) with the presence of fever and purulent vaginal discharge is important [10].

\section{Retained products of conception (RPOC)}

RPOCs complicate approximately $1 \%$ of all deliveries, but are more common after termination of pregnancy or miscarriage [1]. Predisposing factors include the presence of a succenturiate lobe or adhesive placental disorders (placental accreta, increta, percreta), preventing a complete placental delivery [2].

On US, RPOCs have a variable appearance, ranging from an abnormal endometrial-myometrial interface to the presence of heterogeneous echogenic crescentic material $[2,27]$. Focal areas of hyperechogenecity may represent retained placental calcifications [2]. The RPOCs can have internal blood flow, which is often low resistance, and best appreciated at the endometrial-myometrial interface (fig 1d) [2,19].

\section{Ovarian vein thrombosis (thrombophlebitis)}

The pathogenesis is postulated to be retrograde propagation of thrombosed myometrial veins draining an infected placenta. It is more common on the right side and after a cesarean section.

US findings may include anechoic to hypoechoic tubular or serpiginous avascular structures in the region of the right adnexa and inferior vena cava, lack of color Doppler flow in the right ovarian vein, and a thrombus extending to the inferior vena cava [1]. The ovary may be enlarged as well [10]. The accurate diagnosis may be limited by US and US may has relatively poor detection rates, because it may not show the entire length of the ovarian vein in most cases secondary to body habitus and overlying bowel gas. CT and MR imaging may be used to demonstrate the thrombosed vein, which is visualized as a dilated tubular retroperitoneal structure with central thrombus [28].

\section{Bladder flap hematoma}

Bladder flap hematomas occur in patients after cesarean sections and usually appear as a solid complex mass between the posterior bladder wall and the anterior wall of the lower uterine segment [29]. These are typically seen well with transabdominal imaging [1].

\section{Uterine dehiscence and rupture}

Uterine rupture is a rare, catastrophic event that often presents with severe abdominal pain. Although it may occur at antepartum, the uterine dehiscence and rupture is a complication of vaginal delivery, most commonly observed in women who have had a prior cesarean section [2]. Uterine rupture may be limited to dehiscence of the ends of the cesarean scar with an intact overlying serosal layer of the uterine wall. This type of dehiscence does not involve an extrusion of fetal parts into the peritoneal cavity, and therefore results in minimal vaginal bleeding or intraperitoneal hemorrhage. Conversely, full-thickness uterine rupture, with direct communication of the uterine and peritoneal cavities, results in massive hemoperitoneum and carries high fetal and maternal morbidity and mortality rates [2].

Using a rapid assessment with US, the immediate surgery may be necessary for lifesaving. Reported US findings of a uterine rupture include identification of the protruding portion of the amniotic sac, an endometrial or myometrial defect, an extrauterine hematoma, a bulky empty uterus and hemoperitoneum $[2,14]$. 


\section{Conclusion}

Acute pelvic pains in the pregnant and postpartum period are broad-spectrum, ranging from complicated corpus luteal cysts that require routine follow-up to uterine rupture, requiring urgent surgical management. US is the first-line imaging modality of choice for acute pelvic pain. The referral for $\mathrm{CT}$ or $\mathrm{MR}$ imaging should be considered if the US is nondiagnostic. Familiarity with the clinical setting and imaging features of acute pelvic pain in the pregnant and postpartum period will facilitate prompt and accurate diagnosis and treatment for the well-being of the mother and the fetus as well.

\section{References}

1. Cicchiello LA, Hamper UM, Scoutt LM. Ultrasound evaluation of gynecologic causes of pelvic pain. Obstet Gynecol Clin North Am 2011;38:85-114.

2. Kaakaji Y, Nghiem HV, Nodell C, Winter TC. Sonography of obstetric and gynecologic emergencies: Part I, Obstetric emergencies. AJR Am J Roentgenol 2000;174:641-649.

3. Andreotti RF, Harvey SM. Sonographic evaluation of acute pelvic pain. J Ultrasound Med 2012;31:1713-1718.

4. Dupuis CS, Kim YH. Ultrasonography of adnexal causes of acute pelvic pain in pre-menopausal non-pregnant women. Ultrasonography 2015;34:258-267.

5. Doubilet PM, Benson CB, Bourne T, et al. Diagnostic criteria for nonviable pregnancy early in the first trimester. $\mathrm{N}$ Engl J Med 2013;369:1443-1451.

6. Benson CB, Doubilet PM, Peters HE, Frates MC. Intrauterine fluid with ectopic pregnancy: a reappraisal. J Ultrasound Med 2013;32:389-393.

7. Doubilet PM, Benson CB. Double sac sign and intradecidual sign in early pregnancy: interobserver reliability and frequency of occurrence. J Ultrasound Med 2013;32:1207-1214.

8. Dighe M, Cuevas C, Moshiri M, Dubinsky T, Dogra VS. Sonography in first trimester bleeding. J Clin Ultrasound 2008;36:352-366.

9. Lazebnik N, Lazebnik RS. The role of ultrasound in pregnancy-related emergencies. Radiol Clin North Am 2004;42:315-327.

10. Vandermeer FQ, Wong-You-Cheong JJ. Imaging of acute pelvic pain. Clin Obstet Gynecol 2009;52:2-20.

11. Jung SE, Byun JY, Lee JM, et al. MR imaging of maternal diseases in pregnancy. AJR Am J Roentgenol 2001;177:1293-1300.

12. Mehta TS, Levine D, Beckwith B. Treatment of ectopic pregnancy: is a human chorionic gonadotropin level of 2,000 $\mathrm{mIU} / \mathrm{mL}$ a reasonable threshold? Radiology 1997;205:569-573.

13. Potter AW, Chandrasekhar CA. US and CT evaluation of acute pelvic pain of gynecologic origin in nonpregnant premenopausal patients. Radiographics 2008;28:16451659.

14. Di Salvo DN. Sonographic imaging of maternal complications of pregnancy. J Ultrasound Med 2003;22:69-89.

15. Lin EP, Bhatt S, Dogra VS. Diagnostic clues to ectopic pregnancy. Radiographics 2008;28:1661-1671.

16. Levine D. Ectopic pregnancy. Radiology 2007;245:385397.

17. Barnhart KT, Sammel MD, Gracia CR, Chittams J, Hummel AC, Shaunik A. Risk factors for ectopic pregnancy in women with symptomatic first-trimester pregnancies. Fertil Steril 2006;86:36-43.

18. Barnhart KT. Clinical practice. Ectopic pregnancy. N Engl J Med 2009;361:379-387.

19. Elsayes KM, Trout AT, Friedkin AM, et al. Imaging of the placenta: a multimodality pictorial review. Radiographics 2009;29:1371-1391.

20. Glantz C, Purnell L. Clinical utility of sonography in the diagnosis and treatment of placental abruption. J Ultrasound Med 2002;21:837-840.

21. Park SB, Lee JB. MRI features of ovarian cystic lesions. J Magn Reson Imaging 2014;40:503-515.

22. Delvigne A, Rozenberg S. Epidemiology and prevention of ovarian hyperstimulation syndrome (OHSS): a review. Hum Reprod Update 2002;8:559-577.

23. Chiang G, Levine D. Imaging of adnexal masses in pregnancy. J Ultrasound Med 2004;23:805-819.

24. Eyvazzadeh $\mathrm{AD}$, Levine D. Imaging of pelvic pain in the first trimester of pregnancy. Radiol Clin North Am 2006;44:863-877.

25. Masselli G, Derchi L, McHugo J, et al. Acute abdominal and pelvic pain in pregnancy: ESUR recommendations. Eur Radiol 2013;23:3485-3500.

26. Murase E, Siegelman ES, Outwater EK, Perez-Jaffe LA, Tureck RW. Uterine leiomyomas: histopathologic features, MR imaging findings, differential diagnosis, and treatment. Radiographics 1999;19:1179-1197.

27. Zuckerman J, Levine D, McNicholas MM, et al. Imaging of pelvic postpartum complications. AJR Am J Roentgenol 1997; 168:663-668.

28. Virmani V, Kaza R, Sadaf A, Fasih N, Fraser-Hill M. U1trasound, computed tomography, and magnetic resonance imaging of ovarian vein thrombosis in obstetrical and nonobstetrical patients. Can Assoc Radiol J 2012;63:109-118.

29. Baker ME, Bowie JD, Killam AP. Sonography of postcesarean-section bladder-flap hematoma. AJR Am J Roentgenol 1985;144:757-759. 